and that relapse would be likely, and were treatment successful. At the same time, most participants believed that the problem described was common among women in the community and many had thought that 'it might not be too bad' to have the problem described. When asked about the 'main problem' of the person described in the vignette, the modal response (48.4\%) was 'low self-esteem'.

Conclusions: Attitudes and beliefs likely to be conducive to low or inappropriate treatment seeking exist among individuals with eating disorders in the community. These need to be targeted in prevention and early intervention programs.

\section{The role of mu-opioid receptors in the pathology of schizophrenia}

\section{T Money, B Dean, E Scarr}

The Mental Health Research Institute, Melbourne, Australia

Background: Decreased [3H]pirenzepine binding to cortical M1 receptors is a consistent finding in subjects with schizophrenia (Crook et al. Am J Psychiatry 2001, 158 918-925), but the mechanisms causing such decreases are unknown. Recently, low levels of cortical M1 receptors have been reported in muopioid receptor knockout mice (Yoo et al. Synapse 2004, 54 72-82), suggesting that receptor has a role in regulating levels of cortical M1 receptors. We have therefore determined levels of cortical mu-opioid receptors in three cohorts $(1=$ controls, $2=$ schizophrenia with normal levels of $[3 \mathrm{H}]$ pirenzepine binding, $3=$ schizophrenia with low levels of $[3 \mathrm{H}]$ pirenzepine binding) to determine if decreased mu-opioid receptors are associated with low levels of M1 receptors in schizophrenia.

Methods: Western blotting with a rabbit antimu-opioid receptor antibody was used to measure the levels of mu-opioid receptor in Brodmann's area (BA) 9 from 20 subjects from each of the three cohorts described above.

Results: There was no significant difference $(P=0.79)$ between levels of mu-opioid receptors in the controls $(0.98 \pm 0.10)$ and either of the two cohorts of subjects with schizophrenia (cohort 3 : $1.01 \pm 0.11$, cohort 2: $1.01 \pm 0.19$ ).

Conclusions: These data suggest that, at least in BA 9, the mu-opioid receptor is not altered in subjects with low levels of $[3 \mathrm{H}]$ pirenzepine binding and probably does not play a direct role in the regulation of the muscarinic M1 receptor in subjects with schizophrenia.

\section{The 21-item Depression Anxiety Stress Scales as a valid routine clinical outcome measure in the private in-patient setting}

\author{
F Ng ${ }^{1,2}$, M Berk ${ }^{2}$, S Campbell ${ }^{3}$, T Callaly' ${ }^{1}$ S Dodd ${ }^{2}$, \\ T Trauer $^{2}$
}

${ }^{1}$ Barwon Health; ${ }^{2}$ The University of Melbourne, Melbourne, Australia; and ${ }^{3}$ Healthscope, Melbourne, Australia

Background: The self-reported 21-item Depression Anxiety Stress Scales (DASS-21) measure and differentiate negative affective states, which is especially meaningful in the acute psychiatric treatment setting. This study aimed to test the validity of DASS- 21 as a routine clinical outcome measure in the private psychiatric in-patient setting.

Methods: The sample consisted of all admissions to a private psychiatric hospital from January 2004 to December 2005. Scores of four routine measures administered at admission and discharge were retrospectively collected. These measures were the clinician-rated Clinical Global Impression Scale (CGI) and Health of the Nation Outcome Scales in its adult or older persons format (HoNOS or HoNOS65+), and the self-reported Mental Health Questionnaire (MHQ-14) and DASS21 . The four measures were compared using correlation statistics, and differences in measure scores from admission to discharge were analyzed by $t$ test.

Results: Of 786 total admissions, there were 337, 328 and 347 fully completed (ie paired admission and discharge) data sets for the DASS-21 depression, anxiety and stress subscales, respectively. All subscales showed significant reductions in mean scores, in the order of $50 \%$, at discharge compared with baseline $(P<0.001)$. All subscales were correlated with the MHQ-14 subscales $(P<0.0001)$. They also related to partially collapsed CGI categories $(P=0.006)$, except for the baseline stress subscale. The total DASS21 correlated with the total HoNOS scores $(r=0.31$, $P<0.0001)$.

Conclusion: The results support the validity of DASS21 as a routine clinical outcome measure in the private in-patient setting.

\section{Pilot study of physical activity in bipolar disorder}

\section{F Ng ${ }^{1,2}$, S Dodd ${ }^{2}$, M Berk ${ }^{2}$}

${ }^{1}$ Barwon Health; and ${ }^{2}$ The University of Melbourne, Melbourne, Australia

Background: Physical activity has shown efficacy in depression and anxiety, but its benefits in bipolar 